\title{
Machine Learning, deep learning and optimization in computer vision
}

\section{Stéphane Canu}

Stéphane Canu, "Machine Learning, deep learning and optimization in computer vision," Proc. SPIE 10338, Thirteenth International Conference on Quality Control by Artificial Vision 2017, 103380N (14 May 2017); doi: $10.1117 / 12.2277468$

SPIE Event: The International Conference on Quality Control by Artificial Vision 2017, 2017, Tokyo, Japan 


\section{Plenary Talk II}

\section{Machine Learning, Deep Learning and Optimization in Computer Vision}

\section{Prof. Stéphane Canu}

INSA de Rouen, Normandie Univ., France

\section{ABSTRACT}

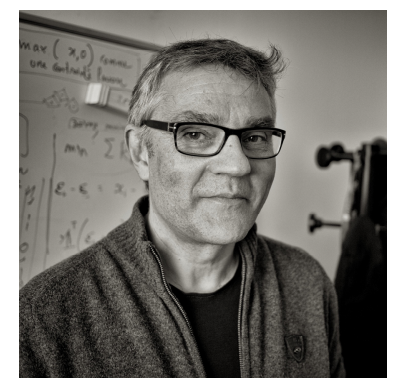

As quoted in the Large Scale Computer Vision Systems NIPS workshop, computer vision is a mature field with a long tradition of research, but recent advances in machine learning, deep learning, representation learning and optimization have provided models with new capabilities to better understand visual content. The presentation will go through these new developments in machine learning covering basic motivations, ideas, models and optimization in deep learning for computer vision, identifying challenges and opportunities. It will focus on issues related with large scale learning that is: high dimensional features, large variety of visual classes, and large number of examples.

\section{BIOGRAPHY}

Stéphane Canu is a Professor of the LITIS Research Laboratory and the Information Technology Department, National Institute of Applied Science, Rouen. He received the Ph.D. degree in system command from the Compiègne University of Technology in 1986. He joined the faculty of the Department of Computer Science, Compiègne University of Technology, in 1987. He received the French Habilitation degree from Paris 6 University. In 1997, he joined the Rouen Applied Sciences National Institute as a Full Professor, where he created the Information Engineering Department. He has been the Dean of this department since 2002, when he was named director of the computing service and facilities unit. In 2004, he joined the Machine Learning Group, ANU/NICTA, Canberra, with A. Smola and B. Williamson. He has published over 100 contriburtions in refereed conference proceedings and 25 papers in journals in the areas of theory, algorithms and applications using kernel machines learning algorithm, and deep learning. His research interests includes kernels machines, deep learning, regularization, model selection, optimization for machine learning and factorization for recommander systems. 